\title{
Generalized Nonlinear Vector Variational-like Inequalitie with set-valued mappings
}

\author{
Ai-hua WANG ${ }^{1}$, Di WU ${ }^{2}$ \\ ${ }^{1}$ College Basic Teaching \& Study Department \\ Bohai University \\ Jinzhou, Liaoning 121013, China \\ Email: wangaihuall@126.com \\ 2 Department of Information Engineering \\ Chaoyang Teachers College \\ Chaoyang, Liaoning 122000, China
}

\author{
Ya-li ZhAO ${ }^{3 *}$ \\ ${ }^{3}$ College of Mathematics and Physics \\ Bohai University \\ Jinzhon, Liaoning 121013, China \\ *Corresponding author Email: yalizhao2000@163.com
}

\begin{abstract}
In this paper, we introduce and study a class of generalized nonlinear vector variational-like inequalities. By utilizing maximal element theorem, we prove the existence of its solutions in the setting of locally convex topological vector space under certain conditions.
\end{abstract}

Keywords-Generalized nonlinear vector variational-like inequalities; Maximal element theorem; Upper semicontinuous; Diagonal convexity

\section{INTRODUCTION}

Variational inequality theory has appeared as an effective and powerful tool to study and investigate a wide class of problems arising in pure and applied sciences including elasticity, optimization, economics, transportation, and structural analysis, see, for instance, [1-3] and the references therein. In 2010, Xiao, Fan and Qi [4] introduced and studied the following class of generalized nonlinear vector variationallike inequalities with set-valued mappings in locally convex topological vector space (locally convex space, in short) and obtained the existence of its solutions.

Let $Z$ be a locally convex space, $E$ be a Hausdorff topological vector space (t.v.s., in short). We denote by $L(E, Z)$ the space of all continuous linear mappings from $E$ into $Z$ and by $\langle l, x\rangle$ the evalution of $l \in L(E, Z)$ at $x \in E$. Let $L(E, Z)$ be a space equipped with $\sigma$-topology. By the corollary of Schaefer ([5], P.80), $L(E, Z)$ becomes a locally convex space. By Ding and Tarafdar [6], the bilinear mapping $\langle.,\rangle:. L(E, Z) \times Z \rightarrow Z$ is continuous. Let $K$ be a nonempty convex subset of a Hausdorff t.v.s. $E, C: K \rightarrow 2^{Z}$ be a set-valued mapping such that int $C(x) \neq \phi$ for all $x \in K, \eta: K \times K \rightarrow E$ be a vectorvalued mapping, let $M, S, T: K \rightarrow 2^{L(E, Z)}$ $N: L(E, Z) \times L(E, Z) \times L(E, Z) \rightarrow 2^{L(E, Z)}$ and $H: K \times K \rightarrow 2^{Z}$ be five set-valued mappings.

This work is supported by the National Natural Science Foundation of China (11371070).
Find $\quad x \in K \quad$ Such that $\exists u \in M(x), v \in S(x)$ and $w \in T(x)$ satisfying

$$
\langle N(u, v, w), \eta(y, x)\rangle+H(x, y) \nsubseteq-\operatorname{int} C(x), \forall y \in K .
$$

In this paper, we introduce and consider the following generalized nonlinear vector variational-like inequalities with set-valued mappings (GNVVLIP, in short): Find $x \in K$ such that

$$
\exists u \in M(x), v \in S(x) \text { and } w \in T(x) \text { satisfying }
$$

$$
\langle N(u, v, w), \eta(y, g(x))\rangle+H(g(x), y) \nsubseteq-\operatorname{int} C(x), \forall y \in K,
$$
where $g: K \rightarrow K$ is a vector-valued mapping. Note that if $g(x)=x$ for each $x \in K$, then the GNVVLIP collapses to the problem introduced and studied by Xiao, Fan and Qi in [4], which includes many known variational inequality problems as special cases, in detail, see [4] and the references therein. So the GNVVLIP is the most general and unifying one, which is also one of the main motivations of this paper.

\section{PReliminaries}

Let int $A$ and $C O A$ denote the interior and convex hull of a set $A$, respectively. Let $X, Y$ be two topological spaces, $\quad T: X \rightarrow 2^{Y} \quad$ be a set-valued mapping and $T^{-1}(y)=\{x \in X: y \in T(x)\} . T$ is said to be upper semicontinuous(u.s.c., in short)if for each $x \in X$ and each open set $V$ in $Y$ with $T(x) \subset V$, there exists an open neighborhood $U$ of $X$ in $X$ such that $T(u) \subset V$ for each $u \in U . T$ is closed if for any net $\left\{x_{\alpha}\right\}$ in $X$ such that $x_{\alpha} \rightarrow X$ and any net $\left\{y_{\alpha}\right\}$ in $Y$ such that $y_{\alpha} \rightarrow y$ and $y_{\alpha} \in T\left(x_{\alpha}\right)$ for each $\alpha$, we have $y \in T(x)$.

Definition 2.1 [7] Let $K$ be a convex subset of a t.u.s. $E$ and $Z$ be a t.u.s.. Let $C: K \rightarrow 2^{Z}$ be a set-valued mapping. 
Assume given any finite subset $\Lambda=\left\{x_{1}, x_{2}, \cdots, x_{n}\right\}$ of $X$, any $x=\sum_{i=1}^{n} \alpha_{i} x_{i} \quad$ with $\alpha_{i} \geq 0$ for $i=1,2, \cdots, n$, and $\sum_{i=1}^{n} \alpha_{i}=1$. Then

(i) a single-valued mapping $f: K \times K \rightarrow Z$ is said to be vector 0-diagonally convex in the second argument if $\sum_{i=1}^{n} \alpha_{i} f\left(x, x_{i}\right) \notin-\operatorname{int} C(x)$.

(ii) a set-valued mapping $f: K \times K \rightarrow Z$ is said to be generalized vector 0 -diagonally convex in the second argument if $\sum_{i=1}^{n} \alpha_{i} f\left(x, x_{i}\right) \nsubseteq-\operatorname{int} C(x)$.

Lemma 2.1 [8] Let $X$ and $Y$ be two topological spaces and $T: X \rightarrow 2^{Y}$ an u.s.c. mapping with compact values. Suppose $\left\{x_{\alpha}\right\}$ is a net in $X$ such that $x_{\alpha} \rightarrow x_{0}$. If $y_{\alpha} \in T\left(x_{\alpha}\right)$ for each $\alpha$, then there exist a $y_{0} \in T\left(x_{0}\right)$ and a subset $\left\{y_{\beta}\right\}$ of $\left\{y_{\alpha}\right\}$ such that $y_{\beta} \rightarrow y_{0}$.

Lemma 2.2 [9] Let $X$ and $Y$ be two topological spaces. If $T: X \rightarrow 2^{Y}$ an u.s.c. set-valued mapping with closed values, then $T$ is closed.

Lemma 2.3 [10] (Maximal Element Theorem) Let $X$ be a nonempty convex subset of a t.v.s. $E$. Let $S: X \rightarrow 2^{X}$ be a setvalued mapping satisfying the following conditions :

(i) $x \notin \operatorname{coS}(x)$ and $S^{-1}$ is open values;

(ii) there exist a nonempty compact subset $A$ of $X$ and a nonempty compact convex subset $B \subset X$ such that for all $x \in X \backslash A$, there exists $Z \in B$ such that $x \in \operatorname{int} S^{-1}(z)$.

Then there exists $x \in X$ such that $S(x)=\phi$.

\section{MAIN RESULTS}

Theorem 3.1 Let $Z$ be a locally convex space, $K$ be a nonempty convex subset of a Hausdorff t.v.s. $E, L(E, Z)$ be a space equipped with $\sigma$-topology, $M, S, T: K \rightarrow 2^{L(E, Z)}$ be three u.s.c. set-valued mappings with nonempty compact values, $g: K \rightarrow K$ be a vector-valued mapping. Suppose that the following conditions are satisfied:

(i) $H$ is generalized vector 0-diagonally convex in the second argument;

(ii) $\eta$ is continuous in both arguments and affine in the first argument with $\eta(x, g(x))=0 . \forall x \in K$;

(iii) $g$ is a continuous and affine mapping;

(iv) for each $y \in K$,

$$
\begin{aligned}
& \langle N(\cdot, \cdot), \eta(y, g(\cdot))\rangle+H(g(\cdot), y): \\
& L(E, Z) \times L(E, Z) \times L(E, Z) \times K \times K \rightarrow 2^{Z}
\end{aligned}
$$

is a upper semicontinuous set-valued mapping with compact values;

(v) $C: K \rightarrow 2^{Z}$ is a set-valued mapping with convex values and the mapping $G(x):=Z \backslash(-$ int $C(x))$ from $K$ into $2^{Z}$ is upper semicontinuous;

(vi) there exist a nonempty compact subset $A$ of $X$ and a nonempty convex subset $B \subset X$ such that for all $x \in X \backslash A, \exists \bar{y} \in B$ such that

$$
\begin{aligned}
& \langle N(u, v, w), \eta(\bar{y}, g(x))\rangle+H(g(x), \bar{y}) \\
& \subseteq-\operatorname{int} C(x), \forall u \in M(x), v \in S(x), w \in T(x) .
\end{aligned}
$$

Then the GNVVLIP has a solution.

Proof. Define a set-valued $P: K \rightarrow 2^{K}$ as follows: For each $X \in K$,

$$
\begin{aligned}
P(x) & =\{y \in K:\langle N(u, v, w), \eta(y, g(x))\rangle+H(G(x), y) \\
& \subseteq-\operatorname{int} C(x), \forall u \in M(x), v \in S(x) \text { and } w \in T(x)\} .
\end{aligned}
$$

First we show that $x \notin \operatorname{coP}(x)$ for all $x \in K$. Suppose to the contrary, there exists $\bar{x} \in K$ such that $\bar{x} \in \operatorname{coP}(\bar{x})$. Then there exist a finite set $\left\{y_{1}, y_{2}, \cdots, y_{n}\right\} \subset P(\bar{x})$ such that $\quad \bar{x} \in \operatorname{co}\left\{y_{1}, y_{2}, \cdots, y_{n}\right\}$, we have $\forall u \in M(x), v \in S(x)$ and $w \in T(x)$,

$$
\begin{aligned}
& \left\langle N(u, v, w), \eta\left(y_{i}, g(\bar{x})\right)\right\rangle+H\left(g(\bar{x}), y_{i}\right) \\
& \subseteq-\operatorname{int} C(\bar{x}), i=1,2, \cdots, n .
\end{aligned}
$$

Since $\operatorname{int} C(\bar{x})$ is a convex set and $\eta$ is affine in the first argument, for $\bar{x}=\sum_{i=1}^{n} t_{i} y_{i} \in K$, where $t_{i} \geq 0, i=1,2, \cdots, n$

with $\sum_{i=1}^{n} t_{i}=1$, we obtain

$$
\begin{aligned}
& \left\langle N(u, v, w), \eta\left(\sum_{i=1}^{n} t_{i} y_{i}, g(\bar{x})\right)\right\rangle+\sum_{i=1}^{n} t_{i} \eta\left(g(\bar{x}), y_{i}\right) \\
& =\langle N(u, v, w), \eta(\bar{x}, g(\bar{x}))\rangle \\
& +\sum_{i=1}^{n} t_{i} \eta\left(g(\bar{x}), y_{i}\right) \subseteq-\operatorname{int} C(\bar{x}) .
\end{aligned}
$$

Since $\eta(\bar{x}, g(\bar{x}))=0 \quad$ by Condition (ii), we get $\sum_{i=1}^{n} t_{i} \eta\left(g(\bar{x}), y_{i}\right) \subseteq-\operatorname{int} C(x)$, Which contradicts Condition (i). And so $x \notin \operatorname{coP}(x)$ for all $x \in K$. Next, we prove that for each $y \in K, P^{-1}(y)$ is a open set in $K$. For this, it is sufficient to prove that the complement 


$$
\left(P^{-1}(y)\right)^{C}=\left\{\begin{array}{l}
x \in K: \\
\{\langle N(u, v, w), \eta(y, g(x))\rangle+H(g(x), y)\} \\
\bigcap Z \backslash(-\operatorname{int} C(x)) \neq \phi,
\end{array}\right.
$$$$
\forall u \in M(x), v \in S(x), w \in T(x)\}
$$

is closed in $K$. In fact, let $\left\{x_{\alpha}\right\}$ be a net in $\left(P^{-1}(y)\right)^{C}$ such that $x_{\alpha} \rightarrow \tilde{x}$. Then there exist $u_{\alpha} \in M\left(x_{\alpha}\right), v_{\alpha} \in S\left(x_{\alpha}\right)$,

$w_{\alpha} \in T\left(x_{\alpha}\right)$ such that

$$
\begin{aligned}
& \left\{\left\langle N\left(u_{\alpha}, v_{\alpha}, w_{\alpha}\right), \eta\left(y, g\left(x_{\alpha}\right)\right)\right\rangle+\eta\left(g\left(x_{\alpha}\right), y\right)\right\} \\
& \bigcap Z \backslash\left(-\operatorname{int} C\left(x_{\alpha}\right)\right) \neq \phi .
\end{aligned}
$$

Since $M, S, T: K \rightarrow 2^{L(E, Z)}$ are three u.s.c. set-valued mappings with compact values, by Lemma $2.1\left\{u_{\alpha}\right\},\left\{v_{\alpha}\right\},\left\{w_{\alpha}\right\}$ have convergent subnets with limits, say, $\tilde{u}, \tilde{v}$, and $, \tilde{w}, \tilde{u} \in M(\tilde{x}), \tilde{v} \in S(\tilde{x}), \tilde{w} \in T(\tilde{x})$.

Without loss of generality, we may assume that $u_{\alpha} \rightarrow \tilde{u}, v_{\alpha} \rightarrow \tilde{v}, w_{\alpha} \rightarrow \tilde{w}$. Suppose that

$$
\begin{aligned}
& z_{\alpha} \in\left\{\left\langle N\left(u_{\alpha}, v_{\alpha}, w_{\alpha}\right), \eta\left(y, g\left(x_{\alpha}\right)\right)\right\rangle+H\left(g\left(x_{\alpha}\right), y\right)\right\} \\
& \cap Z \backslash\left\{-\operatorname{int} C\left(x_{\alpha}\right)\right\} .
\end{aligned}
$$

Since $\langle N(\cdot, \cdot),, \eta(y, g(\cdot))\rangle+H(g(\cdot), y)$ is u.s.c. with compact values, by Lemma 2.1 , there exists a

$$
\tilde{z} \in N(\tilde{u}, \tilde{v}, \tilde{w}), \eta(y, g(\tilde{x}))+H(g(\tilde{x}), y) \quad \text { and } \quad \text { a }
$$
subnet $\left\{z_{\beta}\right\}$ of $\left\{z_{\alpha}\right\}$ such that $z_{\beta} \rightarrow \tilde{z}$. On the other hand, since $G(x)=Z \backslash\{-\operatorname{int} C(x)\}$ is u.s.c. with closed values, which follows Lemma 2.2 that $\tilde{Z} \in Z \backslash\{-\operatorname{int} C(\tilde{x})\}$. hence

$$
\{\langle N(\tilde{u}, \tilde{v}, \tilde{w}), \eta(y, G(\tilde{x}))\rangle+H(g(\tilde{x}), y)\} \cap Z \backslash\{-\operatorname{int} C(\tilde{x})\} \neq \phi .
$$

Thus $\left(P^{-1}(y)\right)^{C}$ is closed in $K$, meaning that $P^{-1}(y)$ is open for each $y \in K$. Furthermore, by Condition (vi), we assert that $\forall x \in K \backslash A, \exists \bar{y} \in B$ such that $x \in \operatorname{int} P^{-1}(\bar{y})$. Hence, $P$ satisfies all the conditions of Lemma 2.3. It follows that there exists an $x \in K$ such that $\exists u \in M(x), v \in S(x)$ and $w \in T(x)$ satisfying

$\langle N(u, v, w), \eta(y, g(x))\rangle+H(g(x), y) \nsubseteq-\operatorname{int} C(x), \forall y \in K$. This completes the proof.

If $N, H$ reduce to be single-valued mappings, then we have the following result.

Corollary 3.1 Let $Z, K, L(E, Z), M, S, T, g$ be same as in Theorem 3.1.Let $N: L(E, Z) \times L(E, Z) \times L(E, Z) \rightarrow L(E, Z)$ and $H: K \times K \rightarrow Z$ be two single-valued mappings. Suppose that the following conditions are satisfied:

(i) $H$ is vector 0 -diagonally convex in the second argument;

(ii) $\eta: K \times K \rightarrow E$ is continuous in both arguments and affine in the first argument with $\eta(x, g(x))=0, \forall x \in K$;

(iii) $g$ is continuous and affine;

(iv) $C: K \rightarrow 2^{Z}$ is a set-valued mapping with convex values and $Z \backslash(-\operatorname{int} C(x))$ is an upper semicontinuous mapping;

(vi) there exist a nonempty compact subset $A$ of $K$ and a nonempty compact convex subset $B$ of $K$ such that $\forall x \in K \backslash A$, $\exists \bar{y} \in B$ such that

$$
\begin{gathered}
\langle N(u, v, w), \eta(\bar{y}, g(x))\rangle+H(g(x), \bar{y}) \in-\operatorname{int} C(x), \forall \\
u \in M(x), v \in S(x) \text { and } w \in T(x) .
\end{gathered}
$$

Then there exists an $x \in K$ Such that $\exists u \in M(x), v \in S(x)$ and,$w \in T(x)$ Satisfying

$$
\langle N(u, v, w), \eta(y, g(x))\rangle+H(g(x), y) \notin-\operatorname{int} C(x), \forall y \in K \text {. }
$$

Remark 3.1 Theorem 3.1 and corollary 3.1 extend the corresponding results of [4].

\section{References}

[1] D. Kinderlehrer, G. Stampacchia. An Introduction to Variational Inequalities and Their applications, in: pure and Applied Mathematics,vol.88, Academic Press, New York, 1980.

[2] X. P. Ding, K. K. Tan. A minimax Inequality with Applications to existence of equilibrium point and fixed point Theorems, Colloquium Mathematicum63(2) (1992) 233-247.

[3] Q. H. Ansari, J. C. Yao. On nondifferentiable and nonconvex vector optimization problems. JOTA, 106(3) (2000)487-500.

[4] G. Xiao, Z. Q. Fan, X. G. Qi. Existence Results for generalized nonlinear vector Variational-like inequality with set-valued mapping. Applied Mathematics Letter, 23 (2010) 44-47.

[5] H. H. Schaefer. Topoloqical vector Spaces, in: Graduate Texts in Mathematics, vol.3, Springer, New York, 1980.

[6] X. P. Ding, E. Tarafdar. Generalized Variational-like inequalities with pseudomonotone set-valued mappings. Archiv der Mathematic, 74(2000) 109-120..

[7] Y. Chiang, O. Chadli, J. C. Yao. Generalized vector equilibrium problems with trifunctions. Journal of Global Optimization, 30 (2004) 135-154.

[8] C. H. Su, V. M. Sehgal. Some fixed point theorems for condensing multifunctions in locally convex spaces. Proceedings of the National Academy of sciences of United States of America 50(1975)150-154.

[9] J. P. Aubin, I. Ekeland. Applied Nonlinear Analysis. John Wiley\&Sons, Inc, New York, 1984.

[10] L. J. Lin, Z. T. Yu, Q. H. Ansari, L. P. Lai. Fixed point and maximal element theorems with applications to abstract economies and minimax inequalities. Journal of Mathematical Analysis and Applications, 284(2003) 656-61. 